\title{
¿Qué nos dicen las investigaciones en didáctica de las matemáticas sobre la enseñanza de la derivada?'
}

\author{
What does research tell us in mathematics education \\ on the teaching of the derivative?
}

O que as pesquisas nos dizem em educação matemática no ensino do derivado?

Recibido: mayo de 2013

Aceptado: agosto de 2013
Carolina Rojas Celis ${ }^{2}$

Edgar Alberto Guacaneme Suárez ${ }^{3}$

\section{Resumen}

Como fragmento del trabajo de grado de la Maestría en Docencia de la Matemática en la Universidad Pedagógica Nacional, se presenta una descripción y clasificación de investigaciones sobre la enseñanza de la derivada realizadas en los campos de la Educación Matemática y de la Educación del Profesor de Matemáticas, para lo cual se emplean en los enfoques descritos por Font (2002) y por Guacaneme \& Mora (2013), respectivamente, con el propósito de determinar qué enfoques están siendo usados en las investigaciones realizadas en los campos. A través de esta clasificación se evidenció una tendencia por los enfoques sistémico y semiótico y escasos trabajos en enfoques socioculturales.

Palabras clave: Profesor; Formación de profesores; Educación Matemática; Matemáticas Escolares; Cálculo; Derivación; Enseñanza de la derivada.

\begin{abstract}
As a fragment of the grade of the Master of Teaching of Mathematics at the National Pedagogical University, is a description and classification of research on teaching the derivative made in the fields of Mathematics Education and Mathematics Teacher Education, for which we used in the approaches described by Font (2002) and Guacaneme \& Mora (2013), respectively, in order to determine what approaches are being used in research in the fields. Through this classification showed a tendency for systemic and semiotic approaches and sociocultural approaches scarce jobs.
\end{abstract}

Keywords: Teacher, Teacher Training, Mathematics Education, Mathematics Education, Calculus, derivation; Teaching derivative.

1 Artículo de Investigación.

2 Universidad Pedagógica Nacional, Maestría en Docencia de la Matemática. Bogotá, Colombia. Contacto: carolinarojasc06@gmail.com

3 Universidad Pedagógica Nacional, Maestría en Docencia de la Matemática. Bogotá, Colombia. Contacto: guacaneme@pedagogica.edu.co 


\section{Resumo}

Como um fragmento do grau de Mestre em Ensino de Matemática da Universidade Pedagógica Nacional, é uma descrição e classificação de pesquisas sobre o ensino do derivado feito nas áreas de Educação Matemática e Professor de Educação Matemática , para o qual foi utilizado nos métodos descritos por fonte (2002) e Guacamene \& Mora (2013), respectivamente, a fim de determinar quais as abordagens que estão a ser utilizados em pesquisas nos campos. Através desta classificação mostrou uma tendência para abordagens sistêmicas e semiótica e abordagens socioculturais empregos escassos.

Palavras-chave: Professor, Formação de Professores, Educação Matemática, Educação Matemática, Cálculo, derivação; derivado de Ensino.

\section{Presentación del problema}

Este trabajo es un fragmento de la tesis de maestría cuya intención es describir si en cursos sobre la enseñanza del Cálculo en universidades formadoras de profesores de Matemáticas, se enseña aquello que nos muestran las investigaciones en Didáctica de las Matemáticas, específicamente en didáctica la derivada. Para ello, debimos preguntarnos primero qué es lo que nos dicen las investigaciones en Didáctica de las Matemáticas sobre la enseñanza de la derivada, cuya respuesta pretendemos esbozar en este documento.

Sin embargo, antes de exhibir los marcos desde los cuales procuramos tal respuesta, queremos reseñar dos documentos que sustentan nuestro trabajo: la ponencia presentada por la profesora María del Mar Moreno (2005), y la réplica a la misma realizada por María Teresa González (2007). En el primero se realiza una reflexión sobre las reformas y cambios que ha ido teniendo la enseñanza del Cálculo en las universidades y se justifica la necesidad de investigaciones sobre la didáctica en el ámbito del conocimiento del profesor, como motor del proceso de enseñanza y aprendizaje; en él, la autora manifiesta también, que debido a que las investigaciones didácticas se han desarrollado teniendo en cuenta diferentes enfoques teóricos los cuales conceden un peso variable a las dimensiones epistemológica, cognitiva y didáctica, se deja de lado la existencia de un solo paradigma, lo que no facilita la comunicación entre investigadores, intentando cada corriente de investigación mantenerse en su "torre de marfil" (Moreno, 2005, p.83).

\section{Marco de referencia conceptual}

Como marco referencial para la clasificación de los documentos seleccionados, ubicamos dos documentos que realizan una descripción de enfoques para los campos de nuestro interés.

El documento de Font (2002), en el campo de la Educación Matemática, describe siete enfoques (cognitivo, constructivismo radical, constructivismo social, sistémico, antropológico, semiótico y crítico), a través de seis aspectos, a saber:

- Una ontología general: Una teoría de la existencia relativa a la consideración

- Una epistemología general que comprende: una teoría de la naturaleza, génesis y validación del conocimiento subjetivo; una teoría de la naturaleza, génesis y validación del conocimiento objetivo; una teoría del significado y de la verdad, implicada por las teorías sobre el conocimiento subjetivo y el objetivo.

- Una teoría sobre la naturaleza de las matemáticas.

- Una teoría sobre el aprendizaje y la enseñanza, que comprende: una teoría general sobre el aprendizaje (cómo se forma el conocimiento personal); una teoría específica sobre el aprendizaje de las matemáticas (cómo se forma el conocimiento matemático personal); una teoría de la enseñanza (los medios para 
facilitar el aprendizaje); una teoría de la enseñanza de las matemáticas (los medios para facilitar el aprendizaje de las matemáticas).

- Una definición del objeto de investigación de la Didáctica de las Matemáticas.

- Una metodología de investigación.

- Para el campo de la Educación del Profesor de Matemáticas, ubicamos el documento (por publicar) de Guacaneme \& Mora (2013), quienes describen cuatro planos o líneas de investigación:

- La línea/plano de las prácticas profesionales de los profesores de Matemáticas que aborda el estudio de: las acciones que el profesor de Matemáticas lleva a cabo en su desempeño docente; las interacciones en las que el profesor se vincula para promover aprendizajes profesionales en comunidades de práctica; el aprendizaje que el profesor logra a partir del estudio personal/individual sobre asuntos que le conmina su práctica.

- La línea/plano conocimiento profesional del profesor de Matemáticas que se ocupa del estudio de las creencias, visiones y concepciones de los profesores de Matemáticas; los conocimientos, destrezas o competencias de los profesores $y$, las diferentes expresiones de la relación entre teoría y práctica.

- La línea/plano formación de los profesores de Matemáticas que contempla el estudio de: las prácticas docentes que realizan los formadores y los procesos de aprendizaje en que se vinculan los futuros profesores o los profesores en ejercicio en el marco de programas de educación de profesores de Matemáticas y, las tareas que el formador diseña o propone en el marco de los programas de educación de profesores.

- La línea/plano conocimiento profesional del formador de profesores de Matemáticas que incluye el estudio de: las creencias, visiones y concepciones de los formadores de profesores de Matemáticas; los conocimientos, destrezas o competencias de los formadores de profesores; las diferentes expresiones de la relación entre teoría y práctica, el aprendizaje logrado a través de la práctica reflexiva y la reflexión como actividad que promueve el aprendizaje desde/para la práctica de formación de profesores de Matemáticas.

\section{Metodología y análisis de datos}

Durante varios meses se recolectaron 62 documentos sobre la enseñanza de la derivada, encontrados en revistas de Educación Matemática, tesis doctorales y de maestría, así como documentos que profesores reconocidos en el campo nos compartieron para ser analizados. De estos documentos, 45 fueron finalmente seleccionados por su importancia en el campo, por su nivel de citación y por la importancia que el autor de ellos ha tenido en el campo.

Algunos de los documentos fueron categorizados en el campo de la Educación del profesor de Matemáticas, en alguna de las líneas o planos propuestos Guacaneme \& Mora (2013). Los demás documentos, relacionados con el campo de la Educación Matemática, fueron categorizados de acuerdo a los enfoques propuestos por Font (2002).

Tablas 1 y 2

\begin{tabular}{|c|c|c|}
\hline $\begin{array}{c}\text { El campo de la } \\
\text { Educación Matemática }\end{array}$ & & \\
\hline ENFOQUE & NOMBRE DEL DOCUMENTO & REFERENCIA \\
\hline Sistémico & $\begin{array}{l}\text { Sobre el estatus de la noción de derivada: de la } \\
\text { epistemologia de Joseph Louis Lagrange al } \\
\text { diseño de una situación didáctica }\end{array}$ & (Cantoral \& Miron, 2000) \\
\hline Cognitivo & El desarrollo del esquema de Derivada & $\begin{array}{l}\text { (Śńnchez-Matamoros, Llinares } \\
\& \text { García, 2006) }\end{array}$ \\
\hline Semiótico & $\begin{array}{l}\text { Formas de argumentación en el cálculo de la } \\
\text { función derivada de la función } \\
\text { definición de derivadas por limites }\end{array}$ & (Font, 2009) \\
\hline \multicolumn{3}{|l|}{$\begin{array}{l}\text { El campo de la Educación } \\
\text { del profesor de } \\
\text { Matemáticas }\end{array}$} \\
\hline Plano/Línea & NOMBRE DEL DOCUMENTO & REFERENCIA \\
\hline $\begin{array}{l}\text { Conocimiento profesional } \\
\text { del profesor de matemáticas }\end{array}$ & $\begin{array}{l}\text { La derivada como objeto matemático y como } \\
\text { objeto de enseñanza y aprendizaje en } \\
\text { profesores de matemática de Colombia }\end{array}$ & (Badillo, 2003) \\
\hline $\begin{array}{l}\text { Prácticas profesionales del } \\
\text { profesor de matemáticas }\end{array}$ & $\begin{array}{l}\text { El papel del profesor en la Enseñanza de la } \\
\text { derivada }\end{array}$ & (Gavilán, 2006) \\
\hline $\begin{array}{l}\text { Conocimiento profesional } \\
\text { del formador del profesores } \\
\text { de matemáticas }\end{array}$ & $\begin{array}{l}\text { La comprensión de la derivada como objeto } \\
\text { de investigación en didáctica de la } \\
\text { matemática }\end{array}$ & $\begin{array}{l}\text { (Sánchez-Matamoros \& } \\
\text { Llinares, 2008) }\end{array}$ \\
\hline
\end{tabular}

Fuente: Elaboración propia 
A continuación se describiremos con algunos ejemplos los documentos que fueron clasificados en los enfoques propuestos para cada uno de los campos:

Cabe resaltar que haber categorzizado los documentos en los enfoques propuestos por los autores en cada uno de los campos, requirió de un estudio bastante cuidadoso de cada uno de ellos, ya que en varios casos no ubicábamos fácilmente un enfoque en el que quedase clasificado exclusivamente, e incluso para algunos documentos tuvimos duda de si ubicarlos en el campo de la Educación del profesor de Matemáticas o en el campo de la Educación Matemática, debido a que no era clara la intención del trabajo. Un ejemplo claro para esta situación fue el estudio del documento de Badillo (2003) ya que, aunque el interés principal de su investigación es reconocer el conocimiento del profesor en cuanto a la disciplina y a su conocimiento didáctico del concepto de Derivada, cabe pensar en que esta investigación bien podría estar ubicada en el campo de la Educación Matemática en el enfoque cognitivo. Algunos de los documentos demandaron la opinión del mismo autor para ser ubicados.

\section{Conclusiones}

La categorización de los documentos permitió visualizar una tendencia por trabajos de investigación interesados en el conocimiento del estudiante, visto desde su estructura mental, como son los trabajos enfocados desde el constructivismo radical y el enfoque cognitivo. En esta tendencia se reconocen también, varios trabajos en los enfoques sistémico y semiótico, y se evidencia un escaso trabajo en los enfoques socio-epistemológicos y socio-culturales.

En el campo de la Educación del profesor de Matemáticas, se reconoce un gran avance en investigaciones cuya intención es el conocimiento profesional del profesor de Matemáticas, y la formación de los profesores de Matemáticas.

Para finalizar, queremos hacer un llamado a que se realicen investigaciones sobre el tipo de conocimiento que un formador de profesores de Matemáticas debe tener para abordar un curso sobre la enseñanza del
Cálculo, pues quizá de este conocimiento dependa que los profesores en formación logren apropiarse de lo fundamental de las investigaciones didácticas sobre la derivada y otros temas del Cálculo, para que en su momento promuevan en sus prácticas docentes mejores aprendizajes sobre el Cálculo.

\section{Referencias}

Font, V. (2002). Una organización de los programas de investigación en Didáctica de las Matemáticas. Revista EMA, 7(2), 127-170.

Guacaneme, E. \& Mora, L. (2013). Líneas de investigación en el campo: La educación del profesor de matemáticas.

González, M. T. (2007). Réplica a la ponencia El papel de la didáctica en la enseñanza del cálculo: evolución, estado actual y retos futuros de la profesora Mar Moreno. En Camacho, Matías; Flores, Pablo; Bolea, María Pilar (Eds.), Investigación en educación matemática XI. Simposio llevado a cabo en el Simposio de la Sociedad Española de Educación Matemática SEIEM, San Cristóbal de la Laguna, España.

Moreno Moreno. \& María del Mar (2005). El papel de la didáctica en la enseñanza del cálculo: evolución, estado actual y retos futuros. En Maz, Alexander; Gómez, Bernardo; Torralbo, Manuel (Eds.), Simposio llevado a cabo en el Simposio de la Sociedad Española de Educación Matemática SEIEM, Córdoba, España.

Cantoral R. \& Mirón H. (2000). Sobre el estatus de la noción de derivada: de la epistemología de Joseph Louis Lagrange al diseño de una situación didáctica. Revista Latinoamericana de Investigación en Educación Matemática. 3(3), 265-292.

Sánchez-Matamoros, G., Llinares, S. \& García, M. (2006). El desarrollo del esquema de derivada. Investigación Didáctica. 24(1), 85-98. 
Font, V. (2009). Formas de argumentación en el cálculo de la función derivada de la función $\mathrm{y}=\mathrm{x}^{\wedge} 2$ sin usar la definición de derivadas por límites. Revista Iberoamericana de Educación Matemática. 1(18), 15-28.

Badillo, E. (2003). La derivada como objeto matemático y como objeto de enseñanza y aprendizaje en profesores de matemática de Colombia. Universitat Autónoma de Barcelona, Barcelona, España.
Gavilán, J. (2006). El papel del profesor en la enseñanza de la Derivada. Educación Matemática. 18(2), 167-170.

Sánchez-Matamoros, G., Llinares, S. \& García, M. (2008). La comprensión de la derivada como objeto de investigación en didáctica de la matemática. Revista Latinoamericana de Investigación en Educación Matemática. 11(2), 267-296. 